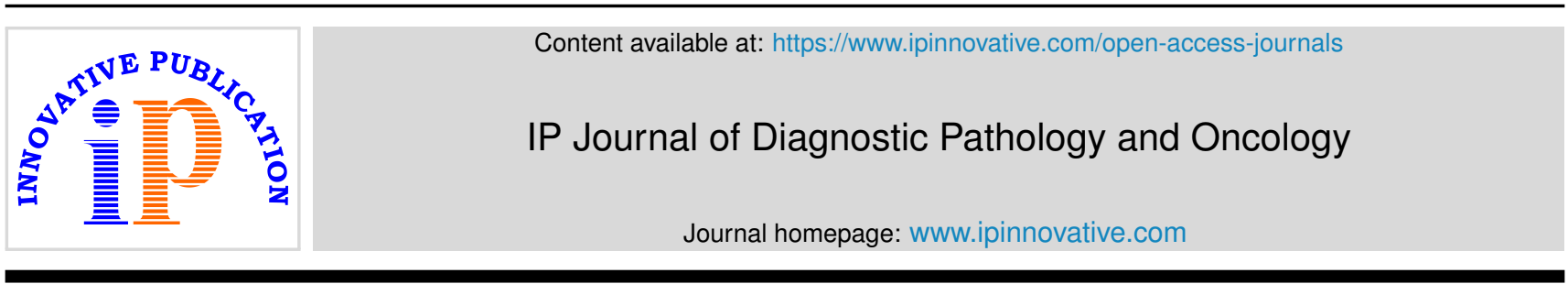

Original Research Article

\title{
Absolute lymphocyte count as a cost effective alternative to CD4 count in HIV positive patients
}

\author{
Amit Nisal $^{1}$, Aaishwarya Dhabe ${ }^{1}$, Anjali Kelkar ${ }^{1} *$, Anu Christopher ${ }^{1}$, \\ Ravindra Nimbargi ${ }^{1}$ \\ ${ }^{1}$ Dept. of Pathology, Bharati Vidyapeeth (Deemed to be University) Medical College, Pune, Maharashtra, India
}

\section{A R T I C L E I N F O}

\section{Article history:}

Received 08-08-2020

Accepted 13-08-2020

Available online 03-09-2020

\section{Keywords:}

Complete blood count

Absolute lymphocyte count

CD4 count

Flow cytometry

\begin{abstract}
A B S T R A C T
Introduction:Acquired immunodeficiency Syndrome (AIDS) is caused by Human Immunodeficiency virus leading to profound immunosuppression and its consequences. Depletion of CD4+ T cells is a hallmark of AIDS apart from anaemia, leucopenia and thrombocytopenia. Monitoring of CD4 count is crucial for an effective treatment. Absolute lymphocyte count (ALC) less than $1000 / \mu 1$ i.e. lymphopenia correlates with lower CD4 counts as per various studies and recommendation by the WHO. Detection of lymphopenia and measurement of ALC are routinely obtained parameters using a basic haematology analyser. This study was done to evaluate the role of absolute lymphocyte count (ALC) as an alternative to CD4 count in HIV positive patients.

Materials and Methods: This prospective observational study was conducted for the period of two years in the department of Pathology of a tertiary care hospital in western India. 110 HIV positive patients consenting to be the part of the study were included. Complete blood counts (CBC), CD4 and CD8 counts were done for all patients following stringent quality control protocols. Statistical analysis was done to evaluate the correlation between various parameters.

Results : $65.5 \%$ cases had lymphopenia with an absolute CD4 count $<200$ cells $/ \mu 1$. Using ROC curve, we found that ALC of less than 575/ $\mu 1$ has a significant statistical association with CD4 count of less than 200/ $\mu$ 1.Correlation of lymphopenia with CD4:CD8 ratio was not found to be statistically significant in our study.

Conclusion : ALC can be considered as a cost effective alternative to absolute CD4 counts in the monitoring of HIV positive patients.
\end{abstract}

(C) 2020 Published by Innovative Publication. This is an open access article under the CC BY-NC license (https://creativecommons.org/licenses/by-nc/4.0/)

\section{Introduction}

Acquired immunodeficiency syndrome (AIDS) is a disease caused by the retrovirus human immunodeficiency virus (HIV) and characterised by immunosuppression leading to opportunistic infections, secondary neoplasms and neurological manifestations. ${ }^{1}$

Profound immunodeficiency, primarily affecting cellmediated immunity, is the hallmark of AIDS. This results chiefly from infection and a severe loss of CD4+T cells as well as impairment in the function of surviving helper $\mathrm{T}$

\footnotetext{
* Corresponding author.

E-mail address: kelkar_anjali@yahoo.com (A. Kelkar).
}

cells. $^{2}$

The common haematological abnormalities seen with HIV include anaemia followed by leucopenia and thrombocytopenia. ${ }^{3}$

The CDC classification system from the revision in the year 1993 combines three categories of the CD4 count with three symptom categories and is closer to a staging system. According to the CD4+ T cells the categories are - Category 1: $>500$ cells $/ \mu 1$ (or CD4\% > 28\%), Category 2: 200-499 cells $/ \mu 1$ (or CD4\% 14\% - 28\%), Category 3: $<200$ cells $/ \mu 1$ (or $\mathrm{CD} 4 \%<14 \%) .{ }^{4}$

Hematologic abnormalities secondary to HIV infection also include venous thromboembolism, hemophagocytic 
syndrome, AIDS - related lymphoma including primary effusion lymphoma, Castleman disease and rarely Hodgkin's disease and myeloma. ${ }^{5}$

In 2018 it was estimated that there are around 37.9 million people living with HIV. ${ }^{6}$ As a priority, antiretroviral therapy (ART) should be initiated in all adults with severe or advanced HIV clinical disease (WHO clinical stage 3 or 4) and adults with CD4 count $\leq 350$ cells $/ \mu 1^{3,7}$

Lymphopenia is defined as absolute lymphocyte count (ALC) $<1000 / \mu 1 .{ }^{8,9}$ In a resource limited country like India, viral load assays as a marker of the disease status are not affordable to majority of the patients infected with HIV. Absolute CD4 counts and CD4: CD8 ratios are used as the surrogate markers to assess the degree of immune deficiency in these circumstances. Flow cytometry is an accepted standard method for determination of absolute counts of CD4 and CD8. ${ }^{10}$

The immune system of patients with HIV infection is characterized by a profound increase in lymphocyte turnover that is immediately reduced with effective antiretroviral therapy. Due to expanding access to HAART (highly active anti-retroviral therapy) in resource limited settings, there has been a need to find out any surrogate marker for CD4 count. Many studies like S. Srirangaraj et al and Agrawal et al found a significant correlation between absolute lymphocyte count (ALC) and CD4 count ( $\mathrm{p}$ value $<0.05)^{11,12}$

CD4 count is measured every 3 months in HIV-1 infected patients. This is done to assess the effectiveness of HAART and to analyse the need for prophylactic treatment for the opportunistic infections. However, in resource limited settings absolute CD4 count is not always available. ${ }^{12}$

In April 2012 WHO recommended that when CD4 cell count is not available, an absolute lymphocyte count of $<1000-1200$ lymphocytes/ $\mu 1$ with stage 2 and 3 disease is an indication to initiate antiretroviral therapy. ${ }^{13}$

\section{Materials and Methods}

A prospective observational study was carried out after taking the institutional ethics committee approval for the period of two years in the department of Pathology of a tertiary care hospital in western India. $110 \mathrm{HIV}$ positive patients consenting to be the part of the study were included. Patients less than 18 years of age and those not willing to undergo testing were excluded from the study.

Whole blood EDTA samples were collected for the tests of $\mathrm{CBC}$ and flow cytometry. CD4 count, CD8 count and CD4:CD8 ratios were carried out on all samples. CBC samples were processed on automated five part differential cell counter (LH-750 from Beckman coulter). CD4 and CD8 counts were obtained by flow cytometry which was done on two lasers six colours flow cytometer (Navios from Beckman Coulter). Chi-square test and Fischer test were used for qualitative data. A two tailed test with $\mathrm{P}$ - value
$<0.05$ was considered as significant. ROC curve was used to find the association between two variables. Anaemia, leucopenia and thrombocytopenia ware graded as per WHO criteria.

Stringent quality control protocols were deployed for complete blood counts and for CD4 and CD8 counts. The quality control data was monitored and immediate corrective actions were taken for any deviations. Satisfactory participation in a national EQA programme was also ensured. The quality control processes ensured accuracy of results. Other relevant patient parameters were obtained from the patients' hospital records and laboratory information system.

\section{Results}

Majority of cases belonged to age group of 31-40 yrs. (47.3\%) followed by $41-50$ yrs. (26.4\%). 65.5\% were males and $34.5 \%$ were females.

Out of 110 cases $60(54.5 \%)$ of them had leucopenia. Out of 110 cases $72(65.5 \%)$ had lymphopenia. 40(36.4\%) had total WBC count $<4000 / \mu 1$ with an absolute CD4 count $<200$ cells $/ \mu$. These values were statistically significant $(\mathrm{p}$ value $=0.014)($ Table 1$)$

The mean CD4 count was 407.85 cells $/ \mu 1.56(51 \%)$ of the patients in our study had CD4 count $<200$ cells $/ \mu 1$. $48(43.6 \%)$ patients with lymphopenia had CD4 values $<200$ cells/microliter and these values were statistically significant ( $\mathrm{p}=<0.001)$ (Figure 1). $63(57.3 \%)$ cases had an ALC $<1000$ cells $/ \mu 1$ with CD4:CD8 ratio $<1$. These values were not statistically significant with a $\mathrm{p}$ value of 0.238 .

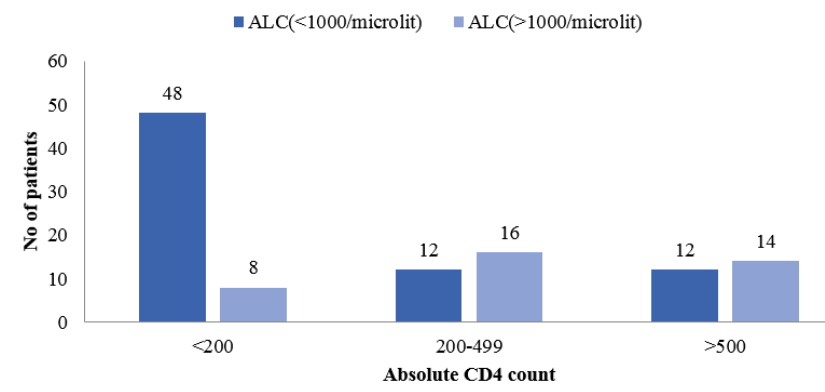

Fig. 1: Correlation of ALC with absolute CD4 count

ROC curve shows trade off between sensitivity (True positivity) and 1- specificity (1- False positivity rate). Classifiers that give curve closer to the left upper corner indicate a better performance. We have used ROC curve to analyse sensitivity of absolute lymphocyte count versus absolute CD4 count.

Using the ROC curve the area under curve is $>90 \%$ when absolute lymphocyte count is $575 / \mu 1$ with a specificity of $90 \%$. This implies that if the absolute lymphocyte count is $<575 / \mu 1$, the probability of CD4 count being $<200 / \mu 1$ are high. (Figure 2). 
Table 1: Correlation of total leucocytes count with CD4 count

\begin{tabular}{ccccc}
\hline Total Leucocytes Count $(/ \mu \mathbf{l})$ & CD4 $(<\mathbf{2 0 0})$ & CD4 (200-499) & CD4 (>500) & P value \\
$<4000$ & 40 & 9 & 13 & 0.014 \\
$4000-11000$ & 11 & 7 & 5 & 5 \\
$>11000$ & 5 & 7 & 12 & 5 \\
\hline
\end{tabular}

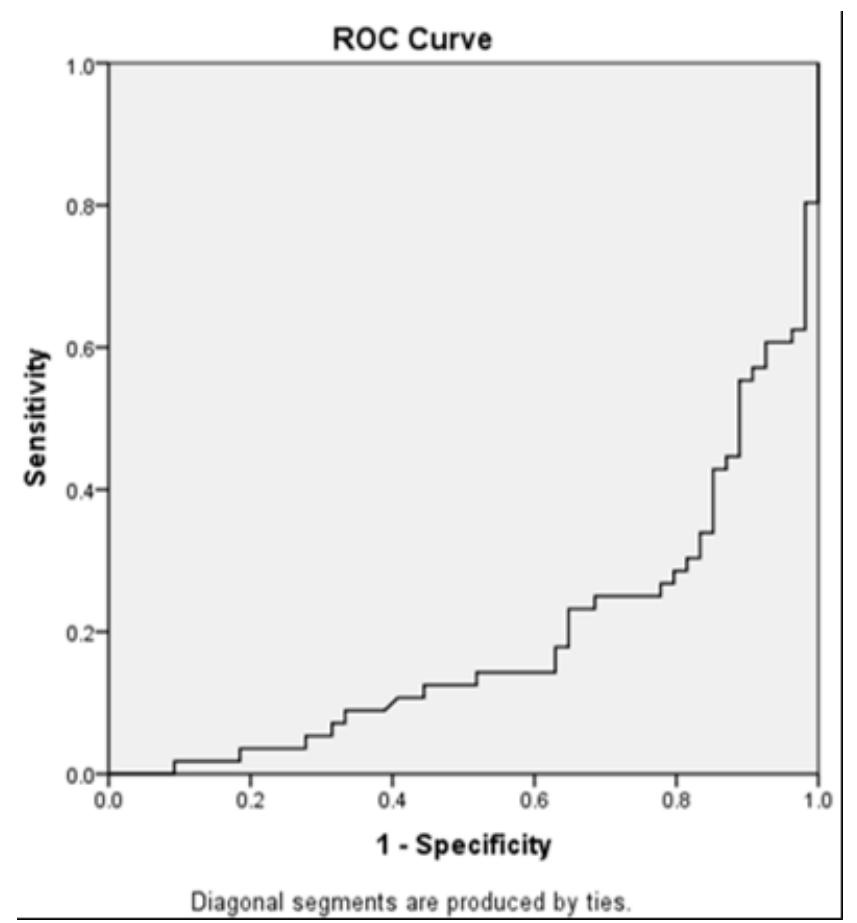

Fig. 2: ROC curve for absolute lymphocyte count and CD4 count

\section{Discussion}

HIV infection is associated with significant immunodeficiency and leads to depletion of CD4+ T cells. Enumeration of CD4 count is routinely used for monitoring of these patients worldwide. However, it requires sophisticated instrument and expertise; making it largely inaccessible. Haematology analysers used in routine laboratories provide absolute lymphocyte count along with the other parameters.

In our study, about $47.3 \%$ cases were in the age group of 31-40 year group. In a study by Shilpa Mittal et al $48 \%$ of patients belong to the age group of 31-40 yrs. Most common age group of 21-40 years with mean age of $36.59 \pm 9.12$ was found in a study by Madhu Balla et al. 1,5

There were $65.5 \%$ males and $34.5 \%$ females which was in concordance with the study by Madhu Balla et al where there were $58 \%$ males and $42 \%$ females. ${ }^{1}$

Leucopenia was seen in $54.5 \%$ patients, whereas in a study done by Erhabor et al and Vijay Kumar et al showed leucopenia in $62 \%$ and $40 \%$ cases respectively. ${ }^{14,15}$ This variation could be due to unavailability of antiretroviral therapy status in our study.
Lymphopenia was found in $65.5 \%$ cases. These findings were in concordance with other studies done by Parinitha et al $(65.2 \%)$ and Treacy et al $(70 \%){ }^{3,16}$

In the present study $51 \%$ cases were found having an absolute CD4 count $<200 / \mu$ l followed by $25.4 \%$ with an absolute CD4 between 200-499/ $\mu$ l and $24 \%$ cases with an absolute CD4 $>500 / \mu \mathrm{l}$; this was in concordance with the study by Attili et al. ${ }^{17}$

In studies by S. Srirangaraj et al and Agrawal et al they found a significant correlation between absolute lymphocyte count and CD4 count with a specificity of $88.9 \%$ and $69.6 \%$ respectively. ${ }^{11,12}$

On using the ROC curve the area under curve is $>90 \%$ when absolute lymphocyte count is $<575 / \mu 1$ with a specificity of $90 \%$. This means that if the absolute lymphocyte count is $<575 / \mu$ l the chances of having a CD4 count $<200 / \mu 1$ were more. Hence a positive correlation between ALC and absolute CD4 count was seen.

\section{Conclusion}

Absolute lymphocyte count obtained on a routine haematology cell counter can be a suitable cost effective alternative for the monitoring of HIV positive patients. This methodology is also accessible to a larger population; flow cytometry being expensive and mostly unavailable. Robust quality control protocols need to be in place for dependable use of this alternative.

\section{Acknowledgment}

$\mathrm{RN}$ was responsible for designing the study. AC and AD performed the tests, basic data collection and preliminary analysis. AN supervised the data analysis and prepared final manuscript. AK did the critical review of the manuscript.

\section{Source of Funding}

None.

\section{Conflicts of Interest}

None.

\section{References}

1. Balla M, Kumar V, Koul KK. A study of haematological manifestations in HIV positive patients-correlation with cd4 count. $J$ Evol Med Dent Sci. 2019;8(19):1545-50.

2. Abbas AK, Aster JC. Robbins and Cotran pathologic basis of disease. Elsevier/Saunders; 2015. 
3. Parinitha SS, Kulkarni MH. Haematological changes in HIV infection with correlation to CD4 cell count. Australas Med J. 2012;5(3):15762.

4. Ward MD, Buehler MJ, Jaffe MH, Berkelman RL. 1993 revised classification system for HIV infection and expanded surveillance case definition for AIDS among adolescents and adults. MMWR Recomm Rep. 1992;41:1-9.

5. Mittal S, Mangal P. Study of anaemia in HIVaids patients and its relation with cd4 counts. Global J Res Anal. 2009;8(5).

6. World Health Organization. Global health sector strategy on HIV 2016-2021. Towards ending AIDS. World Health Organization; 2016.

7. Dubé MP, Stein JH, Aberg JA, Fichtenbaum CJ, Gerber JG, Tashima KT, et al. Adult AIDS Clinical Trials Group Cardiovascular Subcommittee. Guidelines for the evaluation and management of dyslipidemia in human immunodeficiency virus (HIV)-infected adults receiving antiretroviral therapy: recommendations of the HIV Medicine Association of the Infectious Disease Society of America and the Adult AIDS Clinical Trials Group. Clin Infect Dis. 2003;37(5):613-27.

8. Mitrovic Z, Perry AM, Suzumiya J, Armitage JO, Au WY, Coiffier $\mathrm{B}$, et al. The prognostic significance of lymphopenia in peripheral Tcell and natural killer/T-cell lymphomas: A study of 826 cases from the International Peripheral T-cell Lymphoma Project. Am J Hematol. 2012;87(8):790-4.

9. Zidar DA, Al-Kindi SG, Liu Y, Krieger NI, Perzynski AT, Osnard $\mathrm{M}$, et al. Association of lymphopenia with risk of mortality among adults in the US general population. JAMA Netw Open. 2006;2(12):e1916526.

10. Ray K, Gupta SM, Bala M, Muralidhar S, Kumar J. CD4/CD8 lymphocyte counts in healthy, HIV-positive individuals \& AIDS patients. Indian J Med Res. 2006;124(3):319-30.

11. Srirangaraj S, Venkatesha D. Absolute lymphocyte count as a surrogate marker for CD4 counts after six months of HAART initiation in a resource-limited setting in India. Indian J Med Res. 2012;135(6):895.

12. Agrawal PB, Rane SR, Jadhav MV. Absolute lymphocyte count as a surrogate Marker of CD4 count in monitoring HIV infected individuals: A prospective study. J Clin Diagn Res: JCDR. 2016;10(5):EC17.
13. Akinola NO, Olasode O, Adediran IA, Onayemi O, Murainah A, Irinoye O, et al. The Search for a Predictor of CD4 Cell Coun Continues: Total Lymphocyte Count Is Not a Substitute for CD4 Cell Count in the Management of HIV-Infected Individuals in a ResourceLimited Setting. Clin Infect Dis. 2004;39(4):579-81.

14. Erhabor O, Uko EK, Ejele OA, Wachukwu C. Effect of long-term highly active antiretroviral therapy (HAART) on some haematological parameters of HIV; 2019.

15. Kumar V, Jayaswal M. A study of hematologic manifestations in patients with HIV infection. Paripex-Indian J Res. 2019;8(2).

16. Treacy M, Lai L, Costello C, Clark A. Peripheral blood and bone marrow abnormalities in patients with HIV related disease. $\mathrm{Br} J$ Haematol. 1987;65(3):289-94.

17. Attili SV, Singh VP, Rai M, Varma DV, Gulati AK, Sundar S, et al. Hematological profile of HIV patients in relation to immune status-a hospital-based cohort from Varanasi, North India. Turk J Hematol. 2008;25(1):13-9.

\section{Author biography}

Amit Nisal Assistant Professor

Aaishwarya Dhabe Resident

Anjali Kelkar Associate Professor

Anu Christopher Scientific Officer

Ravindra Nimbargi Professor and Head

Cite this article: Nisal A, Dhabe A, Kelkar A, Christopher A, Nimbargi R. Absolute lymphocyte count as a cost effective alternative to CD4 count in HIV positive patients. IP J Diagn Pathol Oncol 2020;5(3):282-285. 